\title{
Study on Reflection Times of Fiber Optic Hydrogen Sensor with Multiple Reflections
}

\author{
Xianling Deng ${ }^{12, a}$, Yike Tang ${ }^{1, b}$, Chuande Zhou ${ }^{2, c}$ and Zelun $\mathrm{Li}^{2, \mathrm{~d}}$
}

${ }^{1}$ College of Mechanical Engineering, Chongqing University, No. 174 Shazhengjie Shapingba District, Chongqing 400044, P.R. China

${ }^{2}$ College of Mechanical and Power Engineering, Chongqing University of Science and Technology,

No. 20 University City East Road Shapingba District, Chongqing 401331, P.R. China

adengxianling2013@126.com, byktang@cqu.edu.cn, 'zcd0013@163.com, d1070141@qq.com,

Keywords: multiple reflections; reflection times; reflective optical fiber sensor; hydrogen

Abstract. Reflection times is the key problem of the reflective optical fiber hydrogen sensor, which directly affects the quality of the sensor sensitivity. In this paper, multiple reflections for reflective optical fiber hydrogen sensor were investigated. Multiple reflection scheme was proposed, the probe structure and parameter relations of multiple reflections were analyzed.

\section{Introduction}

Hydrogen has attracted more and more attention compared to the traditional fuel in recent years, because of its superior characteristics, especially non-pollution. So it has been widely used in many fields, such as aerospace, fuel cells, metal smelting and chemical synthesis. But the hydrogen is volatile, flammable, and explosive, which affect the hydrogen manufacture, storage, transportation, etc. When the hydrogen in the confined space accumulated to a certain concentration, it may be a great threat to human life ${ }^{[1-2]}$. Several kinds of optical fiber hydrogen sensors, such as micro-mirror sensor, evanescent sensor, surface plasmon resonance sensor, acoustic resonator sensor and fiber Bragg grating sensor, have been proposed in recent years ${ }^{[3-5]}$.

\section{Multiple reflection scheme}

Compared with other types of optical fiber sensors, reflective optical fiber hydrogen sensor has many advantages, including strong operability, and low cost. But sensitivity of the reflective optical fiber hydrogen sensor is very urgent. In this work, the method of multiple reflections was proposed.

For this scheme, the beam is needed in the probe multiple reflections. The incident light is no longer perpendicular to the reflector, but there is a certain dip angle. The schematic diagram of the probe is shown in Fig.1

When the beam is incident on the reflector at a certain inclination angle, it passes through the $\mathrm{N}$ times between the two upper and lower reflectors, then coupling in again into optical fiber collimator after reflecting.

The performance of the reflective fiber optic hydrogen sensor is related to the optical parameters of the probe. Further analysis of the optical parameters is needed. According to the structure of the whole sensor and the mechanical structure of the design, the number of reflection is analyzed and determined. 


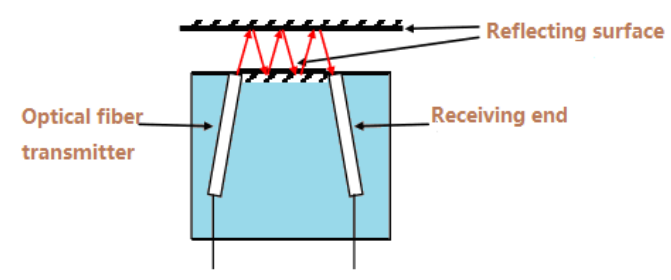

Fig. 1 Schematic diagram of multiple reflecting probes

\section{Analysis of reflection probe}

The reflectivity and reflection number of the reflecting surface of the probe are closely related to the mechanical parameters of the system. The following analysis is based on the mechanical parameters that have been designed. According to the designed structure, the upper and lower limits of incident angle are calculated. The structure of the probe is simplified, and the schematic diagram is shown in Fig. 2(a), focusing on the side of the incident.

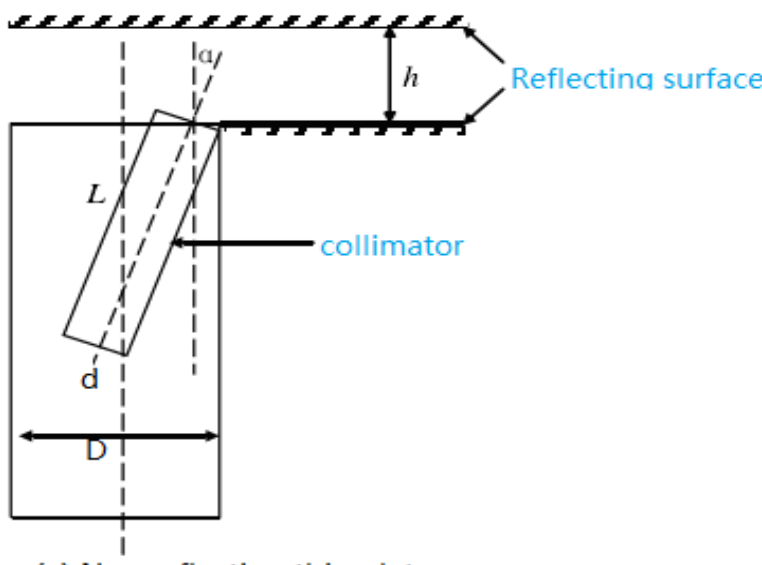

(a) Non reflecting thin plate

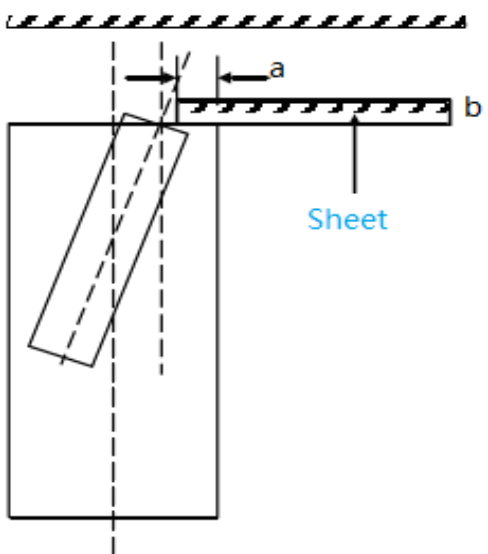

(b) with reflecting thin plate

Fig. 2 Schematic diagram of probe structure section

The following relations can be got according to the geometric relations in Fig. 2(a):

$$
\alpha_{\max }=\arcsin \left(D / \sqrt{L^{2}+d^{2}}\right)-\arctan (d / L) .
$$

$2 h \tan \alpha \geq d / 2$.

Let $\mathrm{L}=20 \mathrm{~mm}, \mathrm{~d}=3.4 \mathrm{~mm}, \mathrm{~h}=4 \mathrm{~mm}$ into Eq.1, the following results can be obtained $\alpha_{\max }=10.5^{\circ}, \alpha \geq 12^{\circ}$. It can be seen that the structural parameters can not satisfy the condition of multiple reflections. The structure needs to be changed. As shown in Fig.2 (b), a sheet with thickness of $\mathrm{Q}$ is used instead of the original reflector, and the left edge of the sheet is not edge align the edges of the hole, but extend the distance from $b$ to the outside. Thus, the relationship between the lower limit of incident angle and the extended distance can be obtained:

$(2 h-b) \tan \alpha \geq d / 2-a$.

\section{Parameter relations of multiple reflections}

In Fig.3, the variation of the lower angle of incidence and the extension distance a are given. 
Among them, $b=1.1 \mathrm{~mm} W h e n$ the extended distance is larger than the collimator radius $\mathrm{d} / 2$, the lower limit of the incident angle is not required. It is necessary to point out that the distance between the upper and lower reflectors is reduced to h-b due to the use of the front surface reflection of the thin plate.

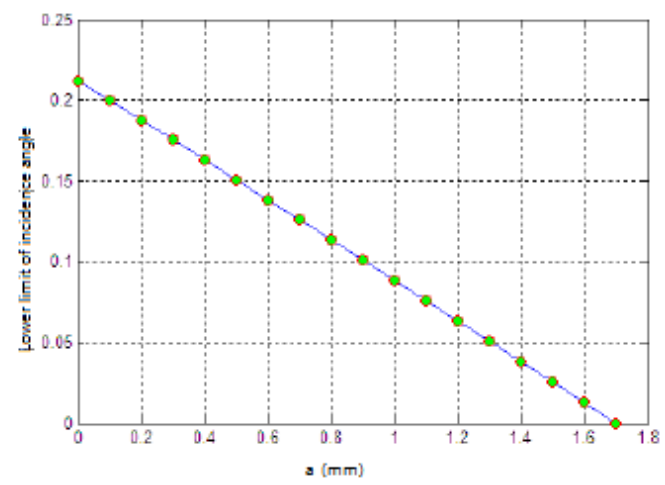

Fig. 3 The relationship between the lower limit of incident angle and ' $a$ '

In order to further determine the extended distance $\mathrm{a}$, the number of reflection times $\mathrm{N}$ and the upper limit of the incident angle, the structure of the probe is simplified by two-dimensional plane. The schematic diagram is shown in Fig.4.

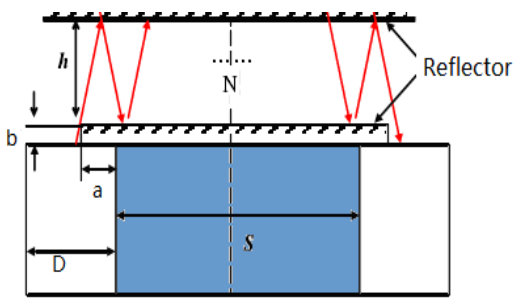

Fig. 4 Structures schematic diagram of multiple reflections

The relationship between the number of reflections, the incident angle and the transverse distance can be obtained by geometric relation:

$$
\begin{aligned}
& 2 \mathrm{~N}(h-a) \tan \alpha \leq s+a . \\
& 2(N+1)(h-b) \tan \alpha \geq s+2 a .
\end{aligned}
$$

The relation between the incident angle and the number of reflections can be obtained by Eq.3. In Fig.5, the change of incident angle with the number of reflection times $\mathrm{N}$ is given at different extension distance a.

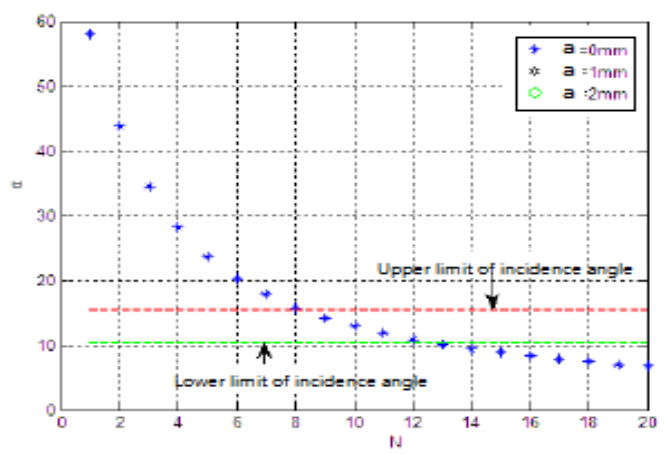

Fig.5 The relationship between the extended distance, the incident angle and $\mathrm{N}$ 
It can be seen from Fig. 5 that the reflection angle is smaller; the change of the reflection angle is more sensitive due to the reflection times. In this design, because the reflectivity of the coating material is limited, the number of reflection can not be very high, so the extension distance can be taken as $\mathrm{p}=1 \mathrm{~mm}$, and the lower limit of the incident angle is $10.2^{\circ}$. The upper limit of the incident angle:

$$
\sin \alpha_{\max }=(D-d / 2) /\left(L-b / \cos \alpha_{\max } .\right.
$$

The upper limit of incident angle can be obtained by solving the equation $\alpha_{m z x}=14.5^{\circ}$. It can be seen that the adjustable range of incident angle has been greatly improved by replacing the original reflector with a thin plate. Under these conditions, the relationship between the number of reflections and the upper and lower angles of incidence is shown in Fig.5. In this condition, the reflection times were $\mathrm{N}=9, \mathrm{~N}=10$ and $\mathrm{N}=11$ by fine-tuning. $\mathrm{N}=9$ and $\mathrm{N}=11$ in the experiment are close to the limits of the inclination angle adjustable, so it is not easy to get. $\mathrm{N}=10$ is relatively easy to adjust, it's the better option.

\section{Conclusion}

In this paper, reflection times for reflective optical fiber hydrogen sensor were investigated. The results show that the reflective optical fiber hydrogen sensor has good sensitivity when the number of reflection times is 10 . The results of this study will play an important role in guiding the following experimental study.

\section{Acknowledgments}

This research is funded by the Scientific and Technological Research Program of Chongqing Municipal Education Commission( Grant No.KJ1501330), the School Research fund of Chongqing University of Science and Technology (Grant No. CK2015Z21), and Chongqing Research Program of Basic Research and Frontier Technology(No.cstc2017jcyjAX0133).

\section{References}

[1] Salehi A and Gholizadeh M. Selectivity Enhancement of MOSFET Hydrogen Sensor With Transparent ITO Gate. Sensors Journal, 2011(11): 1201-1205

[2] Buric M. Multiplexable Low-Temperature Fiber Bragg Grating Hydrogen Sensors . Photonics Technology Letters, 2009 (21): 1594 -1596

[3] S. Sekimoto, H. Nakagawa, S. Okazaki, K. Fukuda, S. Asakura, T. Shige-mori, S. Takahashi, A fiber-optic evanescent-wave hydrogen gas sensor using palladium-supported tungsten oxide, Sens. Actuators B 2000(66), 142-145.

[4] Abdelhamid Boudiba, Pascal Roussel,. Sensing mechanism of hydrogen sensors based on palladium-loaded tungsten oxide (Pd-WO3) .Sensors and Actuators B: Chemical, Volume 187, October 2013, 84-93

[5] Abdelhamid Boudiba, Chao Zhang, Polona Umek. Sensitive and rapid hydrogen sensors based on Pd-WO3 thick films with different morphologies .International Journal of Hydrogen Energy, Volume 38, Issue 5, 19 February 2013, 2565-2577 